\title{
Desempenho de frangos de corte alimentados com dietas contendo diferentes fontes de selênio, zinco e manganês, criados sob condições de estresse térmico
}

\author{
[Performance of broilers fed diets containing different sources of selenium, zinc and manganese \\ reared under thermal stress conditions]
}

\author{
M.M. Boiago ${ }^{1}$, H. Borba ${ }^{2}$, P.A. Souza $a^{2}$, A.M. Scatolini ${ }^{2}$, F.B. Ferrari ${ }^{2}$, A. Giampietro-Ganeco ${ }^{2}$ \\ ${ }^{1}$ Universidade do Estado de Santa Catarina (UDESC / CEO) - Chapecó, SC \\ ${ }^{2}$ Universidade Estadual Paulista "Júlio de Mesquita Filho" - UNESP - Jaboticabal, SP
}

\begin{abstract}
RESUMO
Avaliaram-se o desempenho e o rendimento de carcaça e partes de frangos de corte alimentados com dietas que continham selênio, zinco e manganês complexados ou não a moléculas orgânicas, criados em diferentes temperaturas. Foram utilizados 980 pintinhos machos de um dia de idade, de linhagem comercial, criados durante 42 dias em três câmaras climáticas, que proporcionaram temperaturas alta, termoneutra e baixa. Foi utilizado um delineamento inteiramente ao acaso, em esquema fatorial $3 \times 2+1$, sendo três temperaturas de criação, duas fontes de Se, $\mathrm{Zn}$ e Mn - inorgânica e orgânica, mais o tratamento testemunha - criação em câmara termoneutra sem adição de $\mathrm{Se}$, Zn e Mn na ração, com sete repetições por tratamento. As aves criadas em condições de estresse térmico apresentaram piores resultados para consumo de ração, ganho de peso e viabilidade, sendo mais sensíveis ao calor do que ao frio. Quando criadas em ambientes quentes, a conversão alimentar foi melhor quando se utilizaram os minerais na forma orgânica. Sob temperatura neutra, as aves não necessitaram da suplementação da dieta com os minerais avaliados.
\end{abstract}

Palavras-chave: câmara climática, microminerais orgânicos, rendimento de carcaça

\begin{abstract}
The performance and carcass yield of broilers fed supplemented diets with different selenium, zinc and manganese sources (organic and inorganic) and reared under different environmental temperatures were evaluated. A total of 980 one-day-old broilers were reared until 42 days of age. Three climatic chambers (high, neutral and low temperatures) and twenty chickens per pen, distributed in a $3 x 2+1$ factorial arrangement with three breeding temperatures, two mineral sources and a control treatment that provided neutral temperature without mineral supplementation, with seven replicates each were used. The thermal stressed broilers showed lower results for feed intake, weight gain and viability, mainly under high temperature. The organic mineral utilization provided a better feed conversion ratio when high temperature was tested. Mineral supplementation under neutral breeding temperature was not necessary.
\end{abstract}

Keywords: carcass yield, climatic chambers, organic minerals

\section{INTRODUÇÃO}

A utilização de microminerais na forma orgânica, ou seja, complexados a algum tipo de estrutura orgânica, é hoje bastante estudada, sendo essas fontes apontadas como responsáveis por melhoria dos índices produtivos de frangos de corte (Moreira et al., 2001; Choct et al., 2004).

Quando em situações de estresse térmico, algumas funções metabólicas das aves são

Recebido em 2 de agosto de 2011

Aceito em 25 de agosto de 2012

E-mail: marcel.boiago@udesc.br 
alteradas, na tentativa de se manterem na zona de neutralidade térmica. Entretanto, tais alterações levam à queda de desempenho e do sistema imune das aves (Ribeiro et al., 2008). Diversos estudos mostram a influência negativa do estresse térmico sobre o desempenho de frangos de corte, sendo que o calor é apontado como maior colaborador para tal queda (Laurentz, 2005; Oba, 2012).

A eficácia dos microminerais complexados na nutrição de frangos de corte tem sido demonstrada, na maioria das vezes, por meio de experimentos realizados em condições de temperatura ambiente, sendo poucos os relatos de pesquisadores que submeteram as aves a situações de desafios, como, por exemplo, estresse térmico.
O presente estudo teve o objetivo de avaliar o desempenho e o rendimento de cortes de frangos alimentados com dietas que continham diferentes fontes de selênio, zinco e manganês, criados sob diferentes temperaturas ambientes.

\section{MATERIAL E MÉTODOS}

Foram utilizados 980 pintinhos machos de um dia de idade, da linhagem Cobb, criados durante o período de 42 dias. As aves foram criadas em três câmaras climáticas, equipadas com sistemas de aquecimento e refrigeração, que proporcionaram as diferentes temperaturas (Tab. 1) de acordo com as fases de criação. Foram distribuídas 20 aves por box, com base no peso médio do lote.

Tabela 1. Temperaturas utilizadas durante o período de criação das aves

\begin{tabular}{lccc}
\multicolumn{1}{c}{ Idade (dias) } & \multicolumn{3}{c}{ Temperatura $\left({ }^{\circ} \mathrm{C}\right)$} \\
\cline { 2 - 4 } & Alta & Termoneutra & Baixa \\
\hline $1-3$ & $35 \pm 2$ & $35 \pm 2$ & $35 \pm 2$ \\
$4-7$ & $35 \pm 2$ & $30 \pm 2$ & $25 \pm 2$ \\
$8-14$ & $34 \pm 2$ & $27 \pm 2$ & $20 \pm 2$ \\
$15-21$ & $33 \pm 2$ & $26 \pm 2$ & $18 \pm 2$ \\
$22-42$ & $32 \pm 2$ & $24 \pm 2$ & $16 \pm 2$ \\
\hline
\end{tabular}

Aos 42 dias de idade, foi realizada pesagem das aves e das sobras de ração, objetivando avaliar os índices de desempenho consumo de ração, ganho de peso, conversão alimentar e viabilidade. As rações experimentais (Tab. 2) foram preparadas em um misturador horizontal com capacidade de 75 a $500 \mathrm{~kg}$. As formulações basearam-se nas exigências apresentadas nas Tabelas Brasileiras para Aves e Suínos (Rostagno et al., 2005). As fontes inorgânicas utilizadas foram selenito de sódio, sulfato de zinco e sulfato de manganês, enquanto as "orgânicas" foram Sel-Plex ${ }^{\circledR}$ (Min. $1.000 \mathrm{mg}$ de $\mathrm{Se} / \mathrm{kg}$ do produto), Bioplex ${ }^{\circledR}$ Zinco (Mín. $150.000 \mathrm{mg}$ de $\mathrm{Zn} / \mathrm{kg}$ do produto ) e Bioplex ${ }^{\circledR}$ Manganês (Min. 150.000mg de Mn/kg do produto), da empresa Alltech Agroindustrial do Brasil Ltda.

As quantidades de inclusão dos microminerais nas rações foram 0,$3 ; 60$ e $65 \mathrm{mg}$ de selênio, zinco e manganês por quilo de ração, respectivamente. A pré-mistura vitamínica e mineral utilizada não continha as fontes dos minerais em questão, sendo estas adicionadas separadamente. Assim, o tratamento controle forneceu apenas as concentrações dos minerais contidas no milho e no farelo de soja.

Foi utilizado um delineamento inteiramente ao acaso (DIC), em esquema fatorial $3 \times 2+1$, sendo três temperaturas de criação (alta, termoneutra e baixa), duas fontes de selênio, zinco e manganês (inorgânica e orgânica), mais o tratamento testemunha, onde as aves foram criadas em temperatura neutra sem adição de Se, $\mathrm{Zn}$ e $\mathrm{Mn}$ na ração. Foram utilizadas sete repetições com 20 aves por parcela experimental (10 aves $\left./ \mathrm{m}^{2}\right)$. Após verificação da homogeneidade da variância pelo teste de Bartlett, as médias obtidas foram comparadas pelo teste de Tukey a 5\% de significância, utilizando-se o sistema operacional (Statistical..., 1999). Foram avaliados consumo de ração, ganho de peso, conversão alimentar, viabilidade do lote e rendimento de carcaça e cortes, este último calculado pela relação entre o peso dos cortes (peito, pernas, dorso e asas) e o peso da carcaça, sem o resfriamento em tanque com água e gelo (Chiller), de acordo com Mendes (2001). 
Tabela 2. Composições percentuais e calculadas das rações experimentais de acordo com as fases de criação

\begin{tabular}{lccc}
\hline Ingrediente (\%) & $\begin{array}{c}\text { Inicial } \\
(1-21 \text { dias })\end{array}$ & $\begin{array}{c}\text { Crescimento } \\
(22-35 \text { dias })\end{array}$ & $\begin{array}{c}\text { Acabamento } \\
(36-42 \text { dias })\end{array}$ \\
\hline Milho & 57,20 & 63,89 & 65,04 \\
Farelo de soja & 36,94 & 30,26 & 27,86 \\
Óleo de soja & 1,81 & 2,47 & 3,87 \\
Fosfato bicálcico & 1,83 & 1,63 & 1,38 \\
Calcáreo calcítico & 1,3 & 0,85 & 0,95 \\
Cloreto de sódio & 0,3 & 0,3 & 0,3 \\
Pré-mistura vit. min.* & 0,5 & 0,5 & 0,5 \\
Metionina (98\%) & 0,12 & 0,10 & 0,10 \\
\hline Total & 100 & 100 & 100 \\
\hline Composição calculada & & & 18 \\
\hline Proteína bruta, \% & 21,5 & 19,00 & 3225 \\
Energia metab., kcal/kg & 3000 & 3121 & 0,35 \\
Fósforo disponível, \% & 0,45 & 0,40 & 0,80 \\
Cálcio, \% & 0,95 & 0,84 & 0,75 \\
Metionina + cistina, \% & 0,85 & 0,78 & 0,45 \\
Metionina, \% & 0,50 & 0,46 & 1,00 \\
Lisina, \% & 1,20 & 1,06 & . \\
\hline * Con
\end{tabular}

* Composição do produto (kg) - Inicial: vit. A 176.000UI, vit. D3 40.000UI, vit. E 500mg, vit. K 120mg, vit. B1 $36 \mathrm{mg}$, vit. B2 200mg, vit. B6 70mg, vit. B12 700mcg, niacina $750 \mathrm{mg}$, biotina $3 \mathrm{mg}$, ácido pantotênico $600 \mathrm{mg}$, ácido fólico $30 \mathrm{mg}$, colina $20 \mathrm{mg}$, ferro $1.100 \mathrm{mg}$, cobre $300 \mathrm{mg}$, iodo $24 \mathrm{mg}$, metionina $32 \mathrm{mg}$, cálcio $180 \mathrm{mg}$, fósforo $66 \mathrm{mg}$, sódio $23 \mathrm{mg}$, cloro $36 \mathrm{mg}$, promot. cresc. e efic. alimentar $2 \mathrm{mg}$, coccidiostático $10 \mathrm{~g}$, antifúngico $200 \mathrm{mg}$, antioxidante $1 \mathrm{mg}$, magnésio $5 \mathrm{~g}$, enxofre $4 \mathrm{~g}$, veículo energético e proteico (q.s.p.) 1.000g. Crescimento: vit. A 150.000UI, vit. D3 35.000UI, vit. E 480mg, vit. K 110mg, vit. B1 34mg, vit. B2 170mg, vit. B6 70mg, vit. B12 650mcg, niacina 700mg, biotina $3 \mathrm{mg}$, ácido pantotênico $500 \mathrm{mg}$, ácido fólico $25 \mathrm{mg}$, colina $12 \mathrm{mg}$, ferro $1.100 \mathrm{mg}$, cobre $300 \mathrm{mg}$, iodo $24 \mathrm{mg}$, metionina $20 \mathrm{mg}$, cálcio $176 \mathrm{mg}$, fósforo $60 \mathrm{mg}$, sódio $23 \mathrm{mg}$, cloro $36 \mathrm{mg}$, promot. cresc. e efic. alimentar $2 \mathrm{mg}$, coccidiostático $10 \mathrm{~g}$, antifúngico $200 \mathrm{mg}$, antioxidante $1 \mathrm{mg}$, magnésio $5 \mathrm{~g}$, enxofre $3 \mathrm{~g}$, veículo energético e proteico (q.s.p.) $1.000 \mathrm{~g}$. Acabamento: vit. A $150.000 \mathrm{UI}$, vit. D3 35.000UI, vit. E 450mg, vit. K 100mg, vit. B1 30mg, vit. B2 $160 \mathrm{mg}$, vit. B6 70mg, vit. B12 650mcg, niacina 700mg, biotina 3mg, ácido pantotênico $500 \mathrm{mg}$, ácido fólico $25 \mathrm{mg}$, colina $12 \mathrm{mg}$, ferro $1.100 \mathrm{mg}$, cobre $300 \mathrm{mg}$, iodo $24 \mathrm{mg}$, metionina $18 \mathrm{mg}$, cálcio $176 \mathrm{mg}$, fósforo $58 \mathrm{mg}$, sódio $23 \mathrm{mg}$, cloro $36 \mathrm{mg}$, antifúngico $200 \mathrm{mg}$, antioxidante $1 \mathrm{mg}$, magnésio $5 \mathrm{~g}$, enxofre $3 \mathrm{~g}$, veículo energético e proteico (q.s.p.) $1.000 \mathrm{~g}$.

\section{RESULTADOS E DISCUSSÃO}

Os resultados referentes a consumo de ração, ganho de peso, conversão alimentar e viabilidade das aves são apresentados na Tab. 3. Houve interação significativa para os fatores temperatura de criação versus fonte de microminerais para conversão alimentar, sendo o respectivo desdobramento apresentado na Tab. 4.

Não se observou diferença $(\mathrm{P}>0,05)$ entre o tratamento testemunha e o grupo-tratado, ou seja, as aves que não receberam dietas suplementadas com os microminerais testados apresentaram desempenho similar às demais.

Em relação à fonte dos microminerais utilizados, percebeu-se que a utilização da fonte orgânica ocasionou melhora no ganho de peso e na viabilidade do lote em relação às aves que receberam a fonte inorgânica. Esse resultado diverge dos encontrados por Novelini et al. (2008), que não observaram diferença significativa no desempenho de frangos de corte alimentados com fontes orgânica e inorgânica de selênio. Já Boiago (2006), ao testar fontes inorgânica e orgânica de selênio (selenito de sódio e selenometionina) na ração de frangos de corte da linhagem Cobb, encontrou maior ganho de peso no grupo das aves alimentadas com a fonte orgânica, resultado que corrobora $o$ presente estudo. Segundo Sena e Pedroso (2005), o zinco desempenha diversas funções vitais, sendo uma delas auxiliar no correto funcionamento do sistema imunológico. As aves que receberam os minerais na forma orgânica apresentaram maior viabilidade, resultado que pode ser explicado pela melhor utilização destes na forma orgânica. 
Tabela 3. Resultados obtidos para consumo de ração (CR), ganho de peso (GP), conversão alimentar (CA) e viabilidade das aves no período de um a 42 dias de idade

\begin{tabular}{|c|c|c|c|c|}
\hline \multirow[t]{2}{*}{ Tratamento } & $\begin{array}{l}\text { C R } \\
(\mathrm{kg})\end{array}$ & $\begin{array}{l}\text { G P } \\
(\mathrm{kg})\end{array}$ & $\mathrm{CA}$ & Viabilidade $(\%)$ \\
\hline & \multicolumn{4}{|c|}{ Test. $v s$. fatorial } \\
\hline Testemunha & 5,031 & 2,586 & 1,919 & 91,00 \\
\hline Fatorial & 4,895 & 2,564 & 1,909 & 92,16 \\
\hline Teste F & $2,99^{\mathrm{NS}}$ & $0,18^{\mathrm{NS}}$ & $0,38^{\mathrm{NS}}$ & $0,21^{\mathrm{NS}}$ \\
\hline \multirow[t]{2}{*}{$\operatorname{Pr}>\mathrm{F}$} & 0,109 & 0,682 & 0,550 & 0,652 \\
\hline & \multicolumn{4}{|c|}{ Fontes de microminerais $(\mathrm{F})$} \\
\hline Orgânica & 4,616 & $2,386 \mathrm{~A}$ & 1,947 & $94,33 \mathrm{~A}$ \\
\hline Inorgânica & 4,632 & $2,228 \mathrm{~B}$ & 2,028 & $90,00 \mathrm{~B}$ \\
\hline Teste F & $0,07^{\mathrm{NS}}$ & $5,94 *$ & $13,37 *$ & $4,99 *$ \\
\hline \multirow[t]{2}{*}{$\operatorname{Pr}>\mathrm{F}$} & 0,798 & 0,021 & 0,01 & 0,033 \\
\hline & \multicolumn{4}{|c|}{ Temperatura de criação $(\mathrm{T})$} \\
\hline Alta & $3,825 \mathrm{C}$ & $1,809 \mathrm{~B}$ & 2,089 & $89,00 \mathrm{~B}$ \\
\hline Termoneutra & $4,895 \mathrm{~B}$ & $2,564 \mathrm{~A}$ & 1,909 & $95,50 \mathrm{~A}$ \\
\hline Baixa & $5,152 \mathrm{~A}$ & $2,639 \mathrm{~A}$ & 1,963 & $92,00 \mathrm{AB}$ \\
\hline Teste F & $166,62 * *$ & $174,17 * *$ & $23,35 * *$ & $3,75 *$ \\
\hline $\operatorname{Pr}>\mathrm{F}$ & $<0,01$ & $<0,01$ & $<0,01$ & 0,036 \\
\hline F p/ Int. F X T & $1,38^{\mathrm{NS}}$ & $2,54^{\mathrm{NS}}$ & $6,37 *$ & $1,98^{\mathrm{NS}}$ \\
\hline CV $(\%)$ & 3,68 & 4,63 & 3,05 & 5,77 \\
\hline
\end{tabular}

Na mesma coluna, médias seguidas de letras distintas diferem entre si pelo teste de Tukey $(5 \%)$. $*(\mathrm{P}<0,05)$; ** $(\mathrm{P}<0,01) ; \mathrm{CV}=$ coeficiente de variação. NS = Não significativo.

Observou-se efeito da temperatura sobre todas as características avaliadas $(\mathrm{P}<0,001)$. As aves criadas em temperaturas elevadas apresentaram o menor consumo de ração $(\mathrm{P}<0,05)$, seguidas pelas que foram criadas em temperaturas termoneutra e baixa, respectivamente, que diferiram significativamente entre si. Este resultado se explica pelo fato de as aves, por serem animais homeotermos, terem a capacidade de controlar sua temperatura corporal por meio de ações comportamentais, sendo o controle da ingestão de alimento uma delas. Oba (2012) atribuiu redução da ingestão de alimento por frangos de corte estressados pelo calor ao fato de as aves precisarem aliviar o estresse, que se eleva quando há ingestão de alimento, em razão do incremento metabólico.

O ganho de peso foi menor $(\mathrm{P}<0,05)$ somente nas aves criadas em temperatura quente, sendo similar $(\mathrm{P}>0,05)$ nas aves criadas nas temperaturas neutra e baixa. Essa igualdade no ganho de peso mostrou que houve necessidade de utilização de energia fornecida pela ração para a manutenção da temperatura corporal das aves estressadas pelo frio, já que as aves que foram submetidas a baixas temperaturas apresentaram maior ingestão de ração $(\mathrm{P}<0,01)$.

A viabilidade das aves criadas em temperatura alta foi menor $(\mathrm{P}<0,05)$ que a das criadas em ambiente termoneutro, enquanto aquelas criadas em câmara fria não diferenciaram das demais (P>0,05). Ribeiro et al. (2008) observaram menor consumo de ração ao submeterem frangos de corte ao estresse pelo calor, mas não encontraram efeito sobre a viabilidade do lote, que foi similar à do grupo-controle (ambiente termoneutro), resultado que diverge parcialmente dos obtidos neste estudo.

A Tab. 4 traz o desdobramento da interação entre os fatores temperatura de criação e fonte de microminerais para conversão alimentar (CA) das aves na fase final de criação. 
Tabela 4. Desdobramento da interação entre os fatores temperatura de criação e fonte de microminerais para conversão alimentar (CA) das aves

\begin{tabular}{lccc} 
Fontes de & \multicolumn{3}{c}{ Temperatura de criação } \\
\cline { 2 - 4 } micromineral & Alta & Termoneutra & Baixa \\
\hline Orgânica & $1,995 \mathrm{~B}$ & 1,885 & 1,962 \\
Inorgânica & $2,184 \mathrm{Aa}$ & $1,934 \mathrm{~b}$ & $1,965 \mathrm{~b}$ \\
\hline
\end{tabular}

Médias seguidas por letras distintas minúsculas nas linhas e maiúsculas nas colunas diferem entre si pelo teste de Tukey $(\mathrm{P}<0,05 \%)$.

Não houve diferença significativa entre temperaturas de criação quando se analisaram as aves que receberam rações que continham as fontes orgânicas de microminerais. Entretanto, quando se utilizou a fonte inorgânica, verificouse pior conversão alimentar nas aves criadas em temperaturas elevadas $(\mathrm{P}<0,05)$ em relação àquelas mantidas em temperaturas baixa e termoneutra, que não diferenciaram entre si $(\mathrm{P}>0,05)$. Quanto à temperatura de criação dentro de cada fonte de micromineral, observou-se efeito significativo somente nas aves criadas em temperatura alta, em que a conversão alimentar foi melhor no grupo alimentado com a fonte orgânica $(\mathrm{P}<0,05)$. Nas demais condições térmicas, tal comportamento não foi verificado, ou seja, a conversão foi similar $(\mathrm{P}>0,05)$ nas duas fontes, orgânica e inorgânica. Considerando os resultados acima e o fato de o Brasil apresentar grande variação térmica, com frequentes ondas de calor, pode-se dizer que a utilização das fontes inorgânicas se torna inviável em regiões de calor intenso.

A Tab. 5 contém os valores médios obtidos para rendimentos de carcaça, peito, pernas, dorso e asas das aves submetidas aos diferentes tratamentos. Não houve interação significativa de fonte de micromineral versus temperatura de criação para nenhuma das características avaliadas.

A suplementação da dieta com os microminerais testados não ocasionou alteração $(\mathrm{P}>0,05)$ nos rendimentos dos cortes avaliados. A fonte de microminerais também não afetou $(\mathrm{P}>0,05)$ os rendimentos dos diferentes cortes, ou seja, tanto a utilização da fonte orgânica quanto a da inorgânica levaram a rendimentos semelhantes $(\mathrm{P}>0,05)$.

Oba (2012) avaliou o efeito da utilização de crômio complexado à levedura (Saccharomyces cerevisae) e da temperatura de criação sobre o desempenho e as características da carcaça de frangos de corte abatidos aos 47 dias de idade. Esse autor verificou maior rendimento de carcaça nas aves criadas em temperaturas elevadas, resultado que diverge dos obtidos no presente estudo, em que não se verificou efeito da temperatura $(\mathrm{P}>0,05)$ de criação sobre essa característica.

Percebeu-se influência do fator temperatura de criação sobre os rendimentos de peito, pernas e asas, sendo que o rendimento de peito das aves criadas em temperatura elevada foi mais baixo $(\mathrm{P}<0,05)$ que o das criadas na câmara que proporcionou temperatura neutra, não diferenciando, porém, daquelas submetidas a baixas temperaturas, que também sofreram estresse.

Em relação ao rendimento de pernas, o efeito foi contrário, ou seja, as aves estressadas pelo calor tiveram maior $(\mathrm{P}<0,05)$ porcentagem de pernas em relação às criadas nas demais temperaturas, que não diferiram entre si $(\mathrm{P}>0,05)$. Resultados semelhantes foram relatados por Lana et al. (2000), que, ao avaliarem o efeito da temperatura de criação (alta e termoneutra) sobre o rendimento de cortes de frangos de corte, encontraram diferença significativa entre os tratamentos para rendimentos de pernas, sendo esse maior no grupo estressado pelo calor.

$\mathrm{O}$ rendimento de asas foi maior $(\mathrm{P}<0,05)$ nas aves criadas em temperatura elevada, quando comparadas às criadas em condições termoneutras, e não houve diferença significativa entre estas e as criadas em baixa temperatura, que tiveram rendimentos similares $(\mathrm{P}>0,05)$ aos das aves criadas em temperaturas que proporcionaram conforto térmico. 
Tabela 5. Valores médios obtidos para rendimentos $(\%)$ de carcaça, peito, pernas, dorso e asas das aves abatidas aos 42 dias de idade

\begin{tabular}{|c|c|c|c|c|c|}
\hline \multirow{2}{*}{ Tratamentos } & Carcaça & Peito & Pernas & Dorso & Asas \\
\hline & \multicolumn{5}{|c|}{ Test. $v s$. fatorial } \\
\hline Testemunha & 74,13 & 35,13 & 30,06 & 23,50 & 10,93 \\
\hline Fatorial & 72,54 & 36,91 & 30,04 & 21,85 & 10,60 \\
\hline Teste F & $2,48^{\mathrm{NS}}$ & $4,10^{\mathrm{NS}}$ & $0,01^{\mathrm{NS}}$ & $4,02^{\mathrm{NS}}$ & $1,50^{\mathrm{NS}}$ \\
\hline \multirow{2}{*}{$\operatorname{Pr}>F$} & 0,139 & 0,063 & 0,963 & 0,066 & 0,242 \\
\hline & \multicolumn{5}{|c|}{ Fontesdemicrominerais $(\mathrm{F})$} \\
\hline Orgânica & 72,79 & 35,70 & 30,56 & 21,68 & 10,84 \\
\hline Inorgânica & 72,26 & 35,99 & 30,64 & 21,90 & 10,91 \\
\hline Teste F & $0,80^{\mathrm{NS}}$ & $0,23^{\mathrm{NS}}$ & $0,03^{\mathrm{NS}}$ & $0,13^{\mathrm{NS}}$ & $0,16^{\mathrm{NS}}$ \\
\hline \multirow[t]{2}{*}{$\operatorname{Pr}>F$} & 0,378 & 0,634 & 0,855 & 0,723 & 0,690 \\
\hline & \multicolumn{5}{|c|}{ Temperaturadecriação(T) } \\
\hline Alta & 73,37 & $34,46 \mathrm{~B}$ & $31,75 \mathrm{~A}$ & 21,06 & $11,27 \mathrm{~A}$ \\
\hline Termoneutra & 72,54 & $36,91 \mathrm{~A}$ & $30,04 \mathrm{~B}$ & 21,85 & $10,60 \mathrm{~B}$ \\
\hline Baixa & 71,65 & $36,16 \mathrm{AB}$ & $30,00 \mathrm{~B}$ & 22,47 & $10,77 \mathrm{AB}$ \\
\hline Teste F & $2,80^{\mathrm{NS}}$ & $5,76^{*}$ & $6,85^{*}$ & $1,83^{\mathrm{NS}}$ & $5,26^{*}$ \\
\hline $\operatorname{Pr}>F$ & 0,077 & 0,0080 & 0,003 & 0,178 & 0,011 \\
\hline F p/ Int. F X T & $1,72^{\mathrm{NS}}$ & $0,23^{\mathrm{NS}}$ & $0,42^{\mathrm{NS}}$ & $0,36^{\mathrm{NS}}$ & $2,60^{\mathrm{NS}}$ \\
\hline CV $(\%)$ & 2,23 & 4,63 & 3,95 & 7,52 & 4,38 \\
\hline
\end{tabular}

$\mathrm{Na}$ mesma coluna, médias seguidas de letras distintas diferem entre si pelo teste de Tukey $(5 \%)$. $*(\mathrm{P}<0,05) ; * *$ $(\mathrm{P}<0,01) ; \mathrm{CV}=$ coeficiente de variação. $\mathrm{NS}=$ Não significativo.

\section{CONCLUSÕES}

As aves criadas em condições de estresse térmico apresentaram pior desempenho, sendo mais sensíveis ao calor. A utilização de microminerais complexados a moléculas orgânicas proporcionou melhor desempenho, principalmente quando as aves foram criadas em ambiente quente. Quando criadas em temperatura neutra, as aves não necessitaram da suplementação da dieta com os minerais avaliados.

\section{AGRADECIMENTOS}

À FAPESP (Fundação de Amparo à Pesquisa do Estado de São Paulo), pelo financiamento da pesquisa, e à Alltech do Brasil, pelo fornecimento dos produtos testados.

\section{REFERÊNCIAS}

BOIAGO, M.M. Características produtivas $e$ qualitativas da carne de frangos alimentados com diferentes concentrações e fontes de selênio. 2006. 60f. Dissertação (Mestrado em Zootecnia) - Universidade Estadual Paulista, Faculdade de Ciências Agrárias e veterinárias, Jaboticabal.
CHOCT, M.; NAYLOR, A.J.; REINKE, N. Selenium supplementation affects broiler growth performance, meat yield and feather coverage. Brit. Poult. Sci., v.45, p.677-683, 2004.

LANA, G.R.Q.; ROSTAGNO, H.S.; ALBINO, L.F.T.; LANA, A.M.Q. Efeito da temperatura ambiente e da restrição alimentar sobre o desempenho e a composição da carcaça de frangos de corte. Rev. Bras. Zootec., v.29, p.1117-1123, 2000.

LAURENTZ, A.C. Manejo nutricional das dietas de frangos de corte na tentativa de reduzir a excreção de alguns minerais de importância ambiental. 2005. 131f. Tese (Doutorado em Zootecnia) - Faculdade de Ciências Agrárias e Veterinárias, Universidade Estadual Paulista, Jaboticabal.

MENDES, A.A. Rendimento e qualidade da carcaça de frangos de corte. In: CONFERÊNCIA APINCO DE CIÊNCIA E TECNOLOGIA AVÍCOLAS, 2001, Campinas. Anais... Campinas. 2001. p.79-99. (Resumo). 
MOREIRA, J.; SANTOS, C.D.; PATTO DE ABREU, C.M. et al. Efeito de fonte $\mathrm{e}$ concentrações de Selênio na atividade enzimática da glutathiona peroxidase e no desempenho de frangos de corte. Cienc. Agrotec., v.25, p.661666, 2001.

NOVELINI, L. Desempenho de frangos de corte aos 42 dias suplementados com selênio orgânico na dieta. In: $X$ Encontro da Pósgraduação - UFPEL, 2008. Disponível em: <http://www.ufpel.edu.br/cic/2008/cd/pages/pdf/ CA/CA_01459.pdf>. Acessado em: 05 jan. 2011.

OBA, A.; LOPES, P.C.F.; BOIAGO, M.M. et al. Características produtivas e imunológicas de frangos de corte submetidos a dietas suplementadas com cromo, criados sob diferentes condições de ambiente. Rev. Bras. Zootec., v.41, p.1186-1192, 2012.
RIBEIRO, A.M.; VOGT, L.K.; CANAL, C.W. et al. Suplementação de vitaminas e minerais orgânicos e sua ação sobre a imunocompetência de frangos de corte submetidos a estresse por calor. Rev. Bras. Zootec., v.37, p.636-644, 2008.

ROSTAGNO, H.S.; ALBINO, L.F.T.; DONZELE, J.L. et al. Tabelas brasileiras para aves e suínos: composição dos alimentos $e$ exigências nutricionais. Viçosa: UFV, 2005. p.54 $-56$.

STATISTICAL Analysis Sistem - SAS INSTITUTE. SAS user's guide: statistics. Release 8.02. Cary, 1999.

SENA, K.C.M., PEDROSA, L.F.C. Efeitos da suplementação com zinco sobre o crescimento, sistema imunológico e diabetes. Rev. Nut., v.18, p.251-259, 2005. 